Bulletin de la Société Royale des Sciences, Vol. 90, Articles, 2021, p. 49 - 71

\title{
FONCTIONS CONTINUES MAIS DÉRIVABLES NULLE PART : DE L'EFFROI AU PRINTEMPS DE L'ANALYSE MULTIFRACTALE
}

\author{
LAURENT LOOSVELDT
}

Manuscrit reçu le 12 novembre 2020 et accepté le 11 février 2021

\begin{abstract}
RÉSUMÉ. Après avoir introduit les «monstres » que sont les fonctions continues mais dérivables nulle part, nous présentons brièvement la théorie des espaces de Hölder ponctuels avant de généraliser cette dernière. Quelques résultats fondamentaux sont passés en revue ainsi que des applications de ceux-ci. Nous expliquons notamment comment la transformée en ondelettes amène à des résultats permettant d'estimer la régularité de fonctions. Nous concluons en remarquant que des méthodes numériques, associées aux espaces fonctionnels présentés dans ce papier, permettent de différencier un mouvement brownien d'autres processus.
\end{abstract}

Mots clés : Fonctions non dérivables, Régularité höldérienne, Espace de régularité généralisée, Ondelettes, Formalisme multifractal.

\section{L'EFFROI}

$\mathrm{Au} X I X^{e}$ siècle, la communauté mathématique pensait, de manière quasiment unanime, que toute fonction continue était dérivable partout, sauf éventuellement en quelques points particuliers et isolés, sorte d'erreur de parcours. Une tentative de démonstration de ce fait a même été rédigée par André-Marie Ampère dans [3]. En 1861, lors d'un exposé, Bernhard Riemann fut le premier à contredire cette conjecture en montrant que la fonction

$$
\mathcal{R}: x \mapsto \sum_{j=1}^{+\infty} \frac{\sin \left(j^{2} \pi x\right)}{j^{2}}
$$

est seulement dérivable en les points de la forme $\frac{p}{q}$, où $p$ et $q$ sont deux entiers impairs.

Les bases de l'effroi ultime étaient posées. Celui-ci vint quelques années plus tard, en 1872, lorsque Karl Weierstraß définit, lors d'une conférence à l'Académie de Berlin, toute une famille de fonctions continues sur $\mathbb{R}$ mais dérivables nulle part. Ainsi, si $a \in] 0,1[$ et si $b$ est un nombre impair strictement plus grand que 1 tel que $a b>1+\frac{3}{2} \pi$, la

Cet auteur est soutenu par une bourse d'aspirant FNRS . 
fonction

$$
\mathcal{W}_{a, b}: \mathbb{R} \rightarrow \mathbb{R}: x \mapsto \sum_{j=0}^{+\infty} a^{j} \cos \left(b^{j} \pi x\right)
$$

vérifie cette étrange propriété. La preuve de Weierstraßs peut être lue dans [6]. On peut également remarquer que les conditions sur les paramètres $a$ et $b$ ont été assouplies par Godfrey Harold Hardy dans [19], il suffit d'avoir $a \in] 0,1\left[, b>1\right.$ et $a b>1$ pour s'assurer que $\mathcal{W}_{a, b}$ est continue sur $\mathbb{R}$ tout en n'étant dérivable en aucun point de la droite.
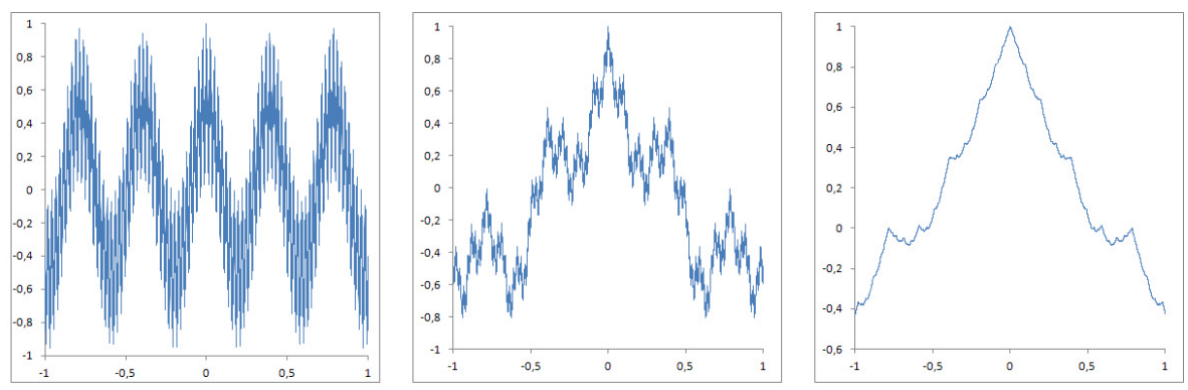

Figure 1. Représentations graphiques de la fonction de Weierstraß avec $a=\frac{1}{2}$ et $b=16$ (à gauche), $b=4$ (au centre) et $b=2^{\frac{4}{3}}$ (à droite).

Henri Poincaré en personne qualifia ces fonctions de « monstres » tandis que Charles Hermite déclara

«Je me détourne avec effroi et horreur de cette plaie lamentable des fonctions continues qui n'ont point de dérivées. »

Fort heureusement, beaucoup de mathématiciens ont bien mieux réagi et nous avons assisté, les années qui suivirent, à un florilège d'exemples de fonctions continues mais dérivables nulle part. Il nous faut mentionner que Bernard Bolzano avait déjà construit en 1830 une telle fonction mais que celle-ci a été publiée quasiment un siècle plus tard, en 1922. Charles Célerier avait également découvert un exemple quelques années avant Weierstraß en 1860, mais celui-ci n'a été publié qu'en 1890.

De nos jours, la situation a bien changé. Avec le temps, les monstres sont devenus de plus en plus courants et, désormais, des fonctions continues mais non dérivables sont observables dans de nombreux phénomènes scientifiques et ce dans des domaines variés allant de la physique [1, 16] à la biologie [2, 42] en passant par la climatologie [13. Le milieu financier regorge également d'exemples de telles fonctions [36, 44].

\section{RÉGUlARITÉ HÖLDÉRIENNE}

Comme souvent en sciences, une fois surmonté l'effroi d'une découverte remettant en doute certains fondements, de nouvelles réflexions se mettent en place amenant à l'émergence de nouvelles théories. 
La dérivabilité était jusqu'alors le meilleur moyen de mesurer la régularité d'une fonction, notamment via les espaces $C_{p}$. Si $\Omega$ est un ouvert de $\mathbb{R}^{d}$ et $p$ un nombre entier naturel non nul, une fonction à valeurs réelles $f \in C_{p}(\Omega)$ si elle est $p$ fois continûment dérivable sur $\Omega$, c'est-à-dire que ses dérivées jusqu'à l'ordre $p$ sont définies et que la dérivée $p$-ème est elle-même une fonction continue. L'espace $C_{0}(\Omega)$ est celui des fonctions continues sur $\Omega$. Ainsi, plus $p$ est grand, plus une fonction appartenant à $C_{p}(\Omega)$ est régulière.

Malheureusement, tous les monstres appartiennent à $C_{0}(\mathbb{R}) \backslash C_{1}(\mathbb{R})$ et les espaces « usuels » ne permettent donc pas de les distinguer. Pour ce faire, il nous faut par conséquent définir des notions intermédiaires à la continuité et la dérivabilité. On va alors s'intéresser au comportement des fonctions en chaque point. Pour illustrer cela, penchons-nous, pour tout $\alpha>0$, sur la fonction

$$
f_{\alpha}: x \mapsto \begin{cases}|x|^{\alpha} \sin \left(\frac{1}{x}\right) & \text { si } x \neq 0 \\ 0 & \text { sinon. }\end{cases}
$$

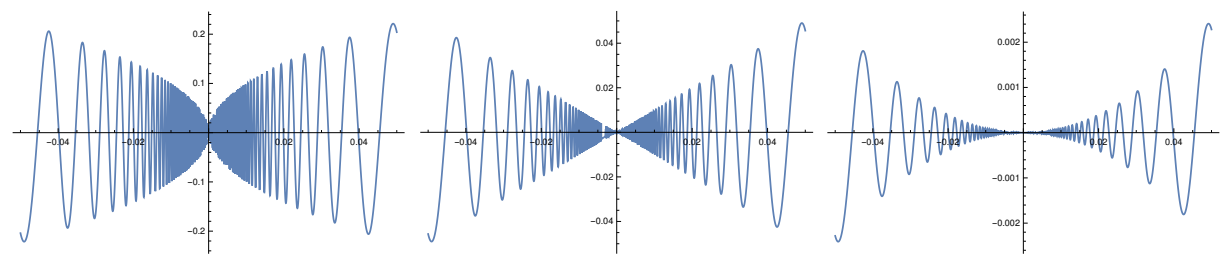

FIGURE 2. La fonction $f_{\alpha}$ pour $\alpha=\frac{1}{2}$, à gauche, $\alpha=1$, au centre, et $\alpha=2$, à droite.

Si $x \neq 0$, il est clair que $f_{\alpha}$ est dérivable en $x$, le seul point problématique est alors 0. Comme le laisse entrevoir la Figure 2, plus $\alpha$ est grand plus l'approche de 0 semble douce. Cela se formalise en remarquant que dès que $\alpha>1, f_{\alpha}$ est dérivable en 0 , étant donné qu'on vérifie sans peine que

$$
\lim _{h \rightarrow 0} \frac{f_{\alpha}(h)-f_{\alpha}(0)}{h}=0,
$$

tandis que, si $\alpha \leq 1$, cette dernière limite n'existe pas et $f_{\alpha}$ n'est pas dérivable en 0 dans ce cas. Rappelons alors que si une fonction réelle $f$ est continue sur un intervalle $[a, b]$ et dérivable sur $] a, b[$, le théorème des accroissements finis garantit que, pour tous $x, y \in[a, b]$, il existe $\left.x_{0} \in\right] x, y\left[\right.$ pour lequel ${ }^{1}$

$$
f(x)-f(y)=(x-y) D f\left(x_{0}\right) .
$$

Ainsi, dès que $D f$ est une fonction bornée sur $[a, b]$, on peut s'assurer, grâce à cette égalité qu'il existe une constante $C$, en l'occurrence ici

1. Suivant les notations d'Euler, $D f$ désigne la dérivée de $f$. 
$C=\sup _{x_{0} \in[a, b]}\left|D f\left(x_{0}\right)\right|$, pour laquelle, pour tous $x, y \in[a, b]$,

$$
|f(x)-f(y)| \leq C|x-y| .
$$

On dit alors $f$ est lipschitzienne sur $[a, b]$. Dans le cadre qui nous occupe, par exemple ${ }^{2}$, on sait déjà que $f_{2}$ est dérivable sur $\mathbb{R}$ et puisque sa dérivée

$$
D f_{2}: x \mapsto \begin{cases}2 x \sin \left(\frac{1}{x}\right)-\cos \left(\frac{1}{x}\right) & \text { si } x \neq 0 \\ 0 & \text { sinon }\end{cases}
$$

est bornée sur tout intervalle borné de $\mathbb{R}, f_{2}$ est lipschitzienne sur ces intervalles. Remarquons cependant que toute fonction lipschitzienne n'est pas dérivable, comme le montre tout simplement la fonction $x \mapsto|x|$, pour laquelle on a bien, pour tout $x \in y \in \mathbb{R},|| x|-| y|| \leq|x-y|$ mais qui n'est pas dérivable en 0. Par ailleurs, il est clair que toute fonction lipschitzienne sur un intervalle est continue sur cet intervalle. Nous avons dès lors introduit une première notion intermédiaire entre la continuité et la dérivabilité. Malheureusement, celle-ci ne nous satisfait pas encore suffisamment pour clore cette caractérisation des fonctions $f_{\alpha}$. En effet, si pour tout $j \in \mathbb{N}$, on définit $x_{j}=(2 \pi j)^{-1}$ et $y_{j}=(2 \pi j+\pi / 2)^{-1}$, on a

$$
\frac{\left|f_{1}\left(x_{j}\right)-f_{1}\left(y_{j}\right)\right|}{\left|x_{j}-y_{j}\right|}=\frac{8 \pi j^{2}+2 \pi j}{2 \pi j+\frac{\pi}{2}}=4 j
$$

et, puisque cette dernière expression tend vers $+\infty$ lorsque $j \rightarrow+\infty$, il est impossible de trouver une constante avec laquelle $f_{1}$ satisferait la condition de Lipschitz sur un intervalle contenant 0. Cependant, si on fixe la valeur de $y$ à 0 dans (1) et qu'on s'intéresse alors exclusivement à $\left|f_{1}(x)-f_{1}(0)\right|$, on obtient, en utilisant simplement le fait que le sinus est borné sur $\mathbb{R}$,

$$
\left|f_{1}(x)-f_{1}(0)\right| \leq|x-0| \text {. }
$$

Ainsi, en adoptant une version ponctuelle de la condition de Lipschitz, nous pouvons enfin donner des informations sur le comportement de $f_{1}$ au voisinage de 0 . Comme ci-dessus, il est clair que, pour tout $0<\alpha<$ 1 , on a

$$
\left|f_{\alpha}(x)-f_{\alpha}(0)\right| \leq|x-0|^{\alpha}
$$

Cette discussion nous amène à vouloir quantifier la régularité ponctuelle des fonctions de $C_{0} \backslash C_{1}$ en tentant de contrôler la vitesse de convergence de $\left|f(x)-f\left(x_{0}\right)\right|$, lorsque $x \rightarrow x_{0}$, par une puissance de $\left|x-x_{0}\right|$. Cela est précisément la raison d'être des espaces de Hölder.

Définition 1. Soient $f: \mathbb{R}^{d} \rightarrow \mathbb{R}$ une fonction localement bornée, $x_{0} \in \mathbb{R}^{d}$ et $0<\alpha \leq 1$. On dit que $f$ appartient à l'espace de Hölder

2. Le choix ici de prendre $\alpha=2$ est dicté par la simplification de l'expression de la dérivée, cette affirmation reste toutefois vérifiée pour tout $\alpha>1$. 
ponctuel d'ordre $\alpha$, en $x_{0}$, ce qu'on note $f \in C^{\alpha}\left(x_{0}\right)$, s'il existe $R>0$ et $C>0$ tels que, pour tout $]^{3} x \in B\left(x_{0}, R\right)$,

$$
\left|f(x)-f\left(x_{0}\right)\right| \leq C\left|x-x_{0}\right|^{\alpha} .
$$

Remarque 2. Dans la définition précédente, si $d>1,\left|x-x_{0}\right|$ désigne la norme euclidienne du vecteur $x-x_{0}$. Notons cependant qu'en dimension finie toutes les normes sont équivalentes et que l'appartenance à un espace de Hölder ponctuel ne sera donc pas affectée par un changement de norme utilisée, seule la valeur de la constante $C$ pourra varier.

Maintenant, rappelons que si $p \in \mathbb{N}^{*}, f \in C_{p}(\Omega)$ et $x_{0} \in \Omega$, la formule de Taylor nous assure l'existence d'un polynôme $P_{x_{0}}$ de degré $p-1$, approximant la fonction $f$ de manière telle que, pour tout $x$ suffisamment proche de $x_{0},\left|f(x)-P_{x_{0}}(x)\right| \leq C\left|x-x_{0}\right|^{p}$. C'est cette propriété d'approximation polynomiale qui est reprise pour définir, pour tout $\alpha>0$, l'espace $C^{\alpha}\left(x_{0}\right)$.

Définition 3. Soient $f: \mathbb{R}^{d} \rightarrow \mathbb{R}$ une fonction localement bornée, $x_{0} \in \mathbb{R}^{d}$ et $\alpha>0$. On dit que $f \in C^{\alpha}\left(x_{0}\right)$ s'il existe $R>0$ et $C>0$ et un polynôme $P_{x_{0}}$ de degré strictement inférieur à $\alpha$ tels que, pour tout $x \in B\left(x_{0}, R\right)$,

$$
\left|f(x)-P_{x_{0}}(x)\right| \leq C\left|x-x_{0}\right|^{\alpha} .
$$

Remarque 4. La définition des espaces de Hölder ponctuels peut subir quelques adaptations, notamment afin de suivre celle donnée dans [45], qui s'attache plus à des espaces fonctionnels.

a) À condition d'autoriser la fonction $f$ à être localement bornée presque partout $\mathrm{A}^{4}$ et ce afin de tirer parti d'espaces fonctionnels classiques, on peut utiliser l'espace fonctionnel $L^{\infty}$. Si $A$ est un sous-ensemble de $\mathbb{R}^{d}$ et si $f$ est localement borné sur $A$, la norme $L^{\infty}$ de $f$ sur $A$ est définie par

$$
\|f\|_{L^{\infty}(A)}:=\inf \{C \geq 0:|f(x)| \leq C \text { pour presque tout } x \in A\} .
$$

On peut alors élargir la définition précédente sous la forme suivante : une fonction $f \in C^{\alpha}\left(x_{0}\right)$ s'il existe $R>0$ et $C>0$ et un polynôme $P_{x_{0}}$ de degré strictement inférieur à $\alpha$ tels que, pour tout $0<r \leq R$,

$$
\left\|f-P_{x_{0}}\right\|_{L^{\infty}\left(B\left(x_{0}, r\right)\right)} \leq C r^{\alpha} .
$$

3. On note $B\left(x_{0}, r\right):=\left\{x \in \mathbb{R}^{d}:\left|x-x_{0}\right|<r\right\}$, pour tout $r>0$.

4. La notion de presque partout se réfère ici à la mesure de Lebesgue utilisée en théorie de l'intégration. Elle généralise la notion de longueur en dimension 1, d'aire en dimension 2 , de volume en dimension $3, \ldots$ L'intégrale d'une fonction sur un ensemble de mesure de Lebesgue nulle vaut toujours zéro. La mesure de Lebesgue fournit une des façons, privilégiée en analyse, d'exprimer l'idée que les points auxquels une certaine propriété n'est pas vérifiée sont « exceptionnels » en s'assurant que la mesure de Lebesgue de l'ensemble de ces points est nulle. 
b) Pour tout $0<r<1$, il existe $j \in \mathbb{N}$ tel que $2^{-(j+1)}<r \leq 2^{-j}$. Il est donc équivalent (voir [11, Lemma 1]) de demander l'existence d'un $J \in \mathbb{N}$, d'une constante $C>0$ et d'un polynôme $P_{x_{0}}$ de degré strictement inférieur à $\alpha$ tels que, pour tout $j \geq J$,

$$
\left\|f-P_{x_{0}}\right\|_{L^{\infty}\left(B\left(x_{0}, 2^{-j}\right)\right)} \leq C 2^{-\alpha j} .
$$

Cela permet de discrétiser le problème de la recherche de l'exposant, ce dont nous tirerons avantage dans les sections suivantes.

c) Définissons pour tout $n \in \mathbb{N}^{*}$ la différence finie d'ordre $n$ et de pas $h \in \mathbb{R}$ de $f$ en $x_{0}$ récursivement par

$$
\Delta_{h}^{1} f\left(x_{0}\right)=f\left(x_{0}+h\right)-f\left(x_{0}\right) \quad \text { et } \quad \Delta_{h}^{n+1} f\left(x_{0}\right)=\Delta_{h}^{1} \Delta_{h}^{n} f\left(x_{0}\right) .
$$

On a alors la formule explicite

$$
\Delta_{h}^{n} f\left(x_{0}\right)=\sum_{j=0}^{n}(-1)^{n-j}\left(\begin{array}{l}
n \\
j
\end{array}\right) f\left(x_{0}+j h\right) .
$$

Grâce au lien existant entre la différence finie et l'approximation polynomiale (voir [7]), l'inégalité (3) est alors équivalente à

$$
\sup _{|h| \leq 2^{-j}}\left\|\Delta_{h}^{\lfloor\alpha\rfloor+1} f\right\|_{L^{\infty}\left(B_{h}\left(x_{0}, 2^{-j}\right)\right)} \leq C 2^{-\alpha j},
$$

où $\lfloor\alpha\rfloor=\max \{n \in \mathbb{Z}: n \leq \alpha\}$ et, pour tout $r>0$,

$$
B_{h}\left(x_{0}, r\right):=\left\{x \in B\left(x_{0}, r\right):[x, x+(\lfloor\alpha\rfloor+1) h] \subseteq B\left(x_{0}, r\right)\right\} .
$$

Le principal avantage de cette définition est que, au moyen de la différence finie, nous pouvons nous contenter de directement travailler sur la fonction $f$ et ses translatées, au lieu de devoir commencer par déterminer un polynôme qui approxime correctement $f$.

Les espaces $C^{\alpha}\left(x_{0}\right)$ sont emboîtés les uns dans les autres ${ }^{5}$, à savoir que si $\alpha<\beta$, alors $C^{\beta}\left(x_{0}\right) \subset C^{\alpha}\left(x_{0}\right)$. Ainsi l'ensemble des $\alpha$ pour lesquels une fonction $f \in C^{\alpha}\left(x_{0}\right)$ est un intervalle $I$ de borne gauche 0 et fermé en ce point. La borne droite de cet intervalle est appelée l'exposant de Hölder de $f$ en $x_{0}$, ce qu'on note $h_{f}\left(x_{0}\right)$. Formellement, on a donc

$$
h_{f}\left(x_{0}\right)=\sup \left\{\alpha \geq 0: f \in C^{\alpha}\left(x_{0}\right)\right\} .
$$

Il est important de remarquer que l'intervalle $I$ peut être fermé en $h_{f}\left(x_{0}\right)$, on dit alors que l'exposant est réalisé, ou ouvert en $h_{f}\left(x_{0}\right)$. Par exemple les fonctions $g_{1}: x \mapsto|x|^{\frac{1}{2}}$ et

$$
g_{2}: x \mapsto \begin{cases}|x|^{\frac{1}{2}} \ln |x| & \text { si } x \neq 0 \\ 0 & \text { sinon. }\end{cases}
$$

ont toutes les deux leur exposant de Hölder en 0 égal à $1 / 2$ mais $g_{1} \in C^{\frac{1}{2}}\left(x_{0}\right)$ tandis que $g_{2} \notin C^{\frac{1}{2}}\left(x_{0}\right)$.

5. Cela se vérifie aisément au moyen de la définition. 
L'exposant de Hölder permet ainsi de quantifier la régularité d'une fonction en un point $x_{0}$ en la situant sur l'échelle des espaces de Hölder. En effet, par définition, si $0<h_{f}\left(x_{0}\right)<\infty$, pour tout $\alpha<h_{f}\left(x_{0}\right)$, $f \in C^{\alpha}\left(x_{0}\right)$ tandis que, pour tout $\beta>h_{f}\left(x_{0}\right), f \notin C^{\beta}\left(x_{0}\right)$.

Grâce à l'exposant de Hölder, on peut donc travailler avec un continuum de valeurs possibles pour déterminer la régularité d'une fonction en un point. Revenons par exemple aux fonctions de Weierstraß. Dans [4, 19], il est montré que, pour tout $x_{0} \in \mathbb{R}$,

$$
h_{\mathcal{W}_{a, b}}\left(x_{0}\right)=-\frac{\log (a)}{\log (b)}
$$

et l'exposant de Hölder nous permet bien de décider, parmi toute cette famille, si une fonction est plus régulière qu'une autre, comme le laissent transparaître les représentations graphiques reprises à la Figure 1.

Pour paraphraser Poincaré, nous avons désormais entre les mains un outil nous permettant de mesurer la dangerosité des monstres que sont les fonctions continues sur $\mathbb{R}$ mais dérivables nulle part. Cela étant, au sens de la régularité höldérienne, la fonction de McCarthy peut ainsi être vue comme faisant partie de l'horreur absolue puisque son exposant de Hölder en tout point est 0 . La fonction de McCarthy $\mathcal{M}$ est construite dans [37] comme ceci : on considère tout d'abord la fonction périodique $g$ définie sur $[-2,2]$ par $g(x)=1-|x|$ et sur $\mathbb{R}$ via $g(x+4)=g(x)$ pour finalement définir

$$
\mathcal{M}: \mathbb{R} \rightarrow[-1,1]: x \mapsto \sum_{j=1}^{+\infty} 2^{-j} g\left(2^{2^{j}} x\right) .
$$

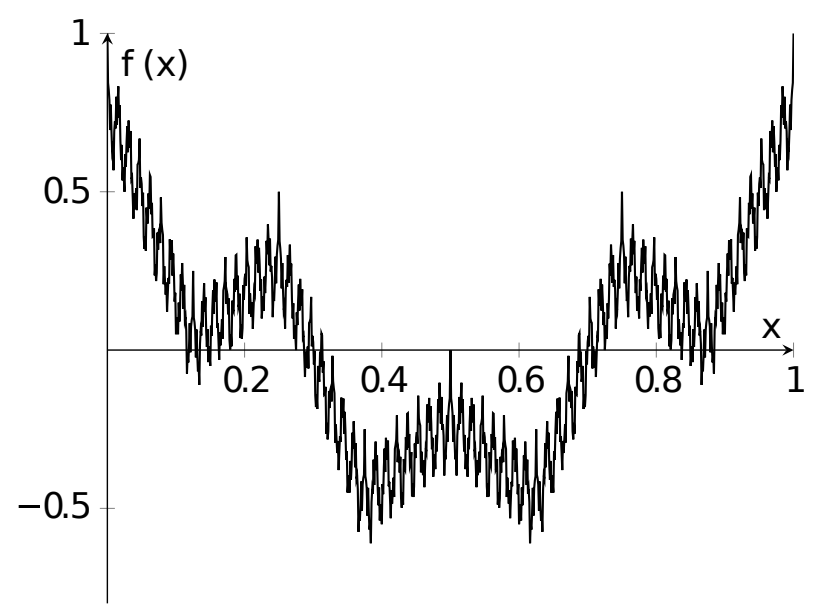

Figure 3. Représentation graphique de la fonction de McCarthy.

Pour s'assurer que, pour tout $x_{0} \in \mathbb{R}, h_{\mathcal{M}}\left(x_{0}\right)=0$, le lecteur intéressé pourra se référer à [40, Proposition VIII.2.8], la démonstration se faisant de manière tout à fait identique, en considérant, en fin de 
raisonnement, pour tout $0<\alpha<1$, le quotient $\frac{\left|f\left(x+h_{j}\right)-f(x)\right|}{\left|h_{j}\right|^{\alpha}}$, au lieu de $\frac{\left|f\left(x+h_{j}\right)-f(x)\right|}{\left|h_{j}\right|}$.

\section{Vers des notions plus fines de RÉGularité}

Parmi les exemples de fonctions continues mais non dérivables, on se doit de citer le mouvement brownien. Celui-ci est notamment utilisé pour modéliser de nombreux phénomènes naturels, comme le mouvement d'une particule dans l'espace, par exemple. Cette fonction doit d'ailleurs son nom au botaniste Robert Brown qui l'utilisa pour décrire la trajectoire des grains de pollen de la Clarkia Pulchella [8]. Albert Einstein en personne s'est longuement intéressé au mouvement brownien (voir [14] pour une réédition d'articles originaux). Nous référons par exemple à [43] pour des applications du mouvement brownien à la chimie moléculaire.

Définition 5. On appelle mouvement brownien sur l'espace probabilisé $(\Omega, \mathcal{B}, \mathbb{P})$ tout processus stochastique gaussien $\left\{B_{t}: t \in \mathbb{R}\right\}$ vérifiant les propriétés suivantes :

- $B_{0}(\omega)=0$ pour $\mathbb{P}$-presque tout $\omega \in \Omega$,

- $t \mapsto B_{t}(\omega)$ est continu pour $\mathbb{P}$-presque tout $\omega \in \Omega$,

- incréments stationnaires $\left.\right|^{6}: B_{t}-B_{s} \sim \mathcal{N}(0,|t-s|)$ pour tous $t, s \in \mathbb{R}$,

- incréments indépendants : $B_{t_{1}}-B_{s_{1}}$ et $B_{t_{2}}-B_{s_{2}}$ sont indépendants pour tous $s_{1}<t_{1} \leq s_{2}<t_{2}$.

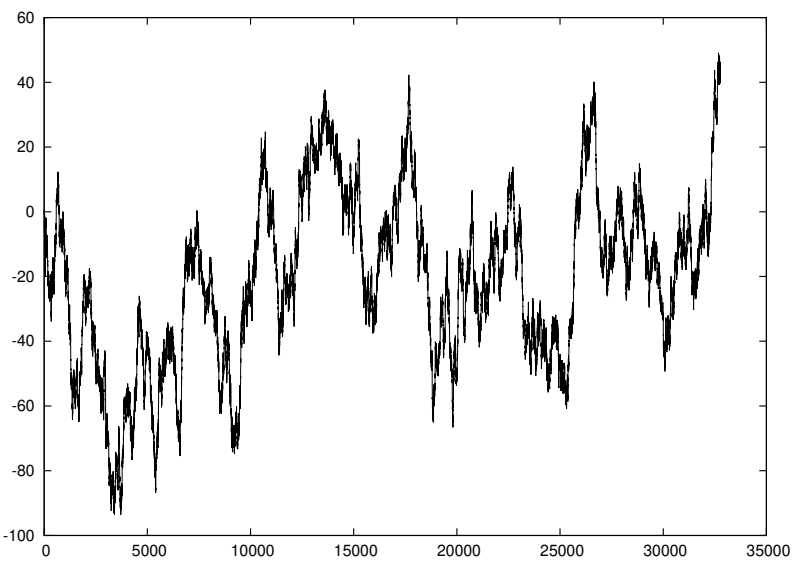

FigURE 4. Une réalisation du mouvement brownien.

En pratique, il est intéressant de savoir déterminer si un signal est un mouvement brownien. En effet, cela indique notamment l'absence de corrélations à longue portée dans le signal (voir par exemple [41, Section

6. Pour rappel, $\mathcal{N}(\mu, \sigma)$ désigne la loi normale de moyenne $\mu$ et d'écart-type $\sigma$. 
3.2]). Or, si on étudie de près la régularité ponctuelle du mouvement brownien, on constate que, pour $\mathbb{P}$-presque tout $\omega \in \Omega$ et pour presque tout $t_{0} \in \mathbb{R}$ (au sens de la mesure de Lebesgue), il existe une constante $C>0$ telle que

$$
\left|B_{t}(\omega)-B_{t_{0}}(\omega)\right| \leq C\left|t_{0}-t\right|^{\frac{1}{2}} \sqrt{\log \log \left|t-t_{0}\right|^{-1}}
$$

alors que, pour tout $t_{0} \in \mathbb{R}, h_{B \text {.( } \omega)}\left(t_{0}\right)=\frac{1}{2}$ ( [25]). Ainsi, les espaces de Hölder et l'exposant associé effacent la correction logarithmique itérée et sont incapables de faire la distinction entre le mouvement brownien et un autre processus dans lequel une telle correction n'apparaît pas. Pour pallier ce manquement, on veut pouvoir définir des espaces de Hölder généralisés, pour lesquels les membres de gauche de (3) et (5) ne sont plus comparés à une suite dyadique 7 mais à des suites plus générales, que nous appelons admissibles.

Définition 6. Une suite $\boldsymbol{\sigma}=\left(\sigma_{j}\right)_{j}$ de nombres réels strictement positifs est une suite admissible s'il existe deux constantes strictement positives $d_{0}$ et $d_{1}$ de sorte que, pour tout $j$,

$$
d_{0} \sigma_{j} \leq \sigma_{j+1} \leq d_{1} \sigma_{j}
$$

L'intérêt de travailler avec des suites admissibles est que, bien qu'elles soient bien plus générales qu'une suite dyadique, on peut facilement obtenir une caractérisation assez précise de leur comportement asymptotique. En effet, si $\boldsymbol{\sigma}$ est une suite admissible et si on définit, pour tout $j$,

$$
\underline{\sigma}_{j}=\inf _{k \in \mathbb{N}} \frac{\sigma_{j+k}}{\sigma_{k}} \quad \text { et } \quad \bar{\sigma}_{j}=\sup _{k \in \mathbb{N}} \frac{\sigma_{j+k}}{\sigma_{k}},
$$

alors les indices de Boyd inférieur et supérieur 8 de $\sigma$

$$
\underline{s}(\boldsymbol{\sigma})=\lim _{j} \frac{\log _{2} \underline{\sigma}_{j}}{j} \text { et } \bar{s}(\boldsymbol{\sigma})=\lim _{j} \frac{\log _{2} \bar{\sigma}_{j}}{j}
$$

sont tels que, pour tout $\varepsilon>0$, il existe $C>0$ tel que, pour tout $j$,

$$
C^{-1} 2^{j(\underline{s}(\boldsymbol{\sigma})-\epsilon)} \leq \underline{\sigma}_{j} \leq \frac{\sigma_{j+k}}{\sigma_{k}} \leq \bar{\sigma}_{j} \leq C 2^{j(\bar{s}(\boldsymbol{\sigma})+\epsilon)} .
$$

Bien évidemment, pour tout $\alpha \in \mathbb{R}$, la suite $\left(2^{\alpha j}\right)_{j}$ est admissible avec $\underline{s}\left(\left(2^{\alpha j}\right)_{j}\right)=\bar{s}\left(\left(2^{\alpha j}\right)_{j}\right)=\alpha$.

En pratique, nous allons travailler avec des suites de la forme $\left(\sigma_{j}=2^{\alpha j} \delta_{j}\right)_{j}$, où $\left(\delta_{j}\right)_{j}$ est une suite admissible asymptotiquement plus faible que la partie dyadique $\left(2^{\alpha j}\right)_{j}$. Par exemple, si $\psi$ est une fonction

7. Une suite dyadique est une suite $\left(x_{j}\right)_{j}$ pour laquelle il existe $\alpha \in \mathbb{R}$ telle que, pour tout $j, x_{j}=2^{\alpha j}$. Les suites dyadiques correspondent en fait précisément aux suites géométriques de raison $q>0$ puisque la fonction $\alpha \mapsto 2^{\alpha}$ est une bijection de $\mathbb{R}$ dans $] 0,+\infty[$.

8. On désigne par $\log _{2}$ le logarithme en base 2 . 
à variation lente, c'est-à-dire une fonction strictement positive pour laquelle, pour tout $r>0$,

$$
\lim _{t \rightarrow 0} \frac{\psi(r t)}{\psi(t)}=1,
$$

la suite $\left(2^{\alpha j} \psi\left(2^{j}\right)\right)_{j}$ est admissible et ses indices de Boyd sont $\alpha$ (voir [28]). Le logarithme est une fonction à variation lente.

Remarque 7. Le lecteur familier avec les questions de comportements ponctuels et uniformes des fonctions pourrait dans la suite de cet article légitiment s'interroger sur le lien qui existe entre les suites admissibles et les modules de continuité. En fait, on peut facilement montrer [27] que si $\boldsymbol{\sigma}=\left(\sigma_{j}\right)_{j}$ est une suite admissible, il existe un module de continuité $\omega$ tel que, pour tout $j, \sigma_{j}=\omega\left(2^{-j}\right)$ si et seulement si $\boldsymbol{\sigma}$ est une suite décroissante. De plus, $\omega$ est continu en 0 si et seulement si $\boldsymbol{\sigma}$ converge vers 0 .

Un autre inconvénient notoire des espaces de Hölder est qu'ils demandent de travailler avec des fonctions localement bornées (presque partout) alors qu'on voudrait pouvoir analyser des exemples ne vérifiant pas cette dernière propriété (voir [23] par exemple). Une possibilité de généralisation, tout en gardant un cadre proche, est d'utiliser les espaces $L^{p}$ et leur norme associée.

Définition 8. Soient $1 \leq p<\infty$ et $A$ un sous-ensemble de $\mathbb{R}^{d}$. Une fonction mesurable $f: A \rightarrow \mathbb{C}$ appartient à l'espace $L^{p}(A)$ si $|f|^{p}$ est intégrable sur $A$. Dans ce cas, la norme $L^{p}(A)$ de $f$ est définie par

$$
\|f\|_{L^{p}(A)}:=\left(\int_{A}|f(x)|^{p} d x\right)^{\frac{1}{p}} .
$$

Le cas $p=\infty$ a déjà été défini lors du premier point de la Remarque 4 .

On dit qu'une fonction $f \in L_{\text {loc }}^{p}\left(\mathbb{R}^{d}\right)$ si, pour tout sous-ensemble compact $K$ de $\mathbb{R}^{d}, f \in L^{p}(K)$.

Remarque 9. Pour être tout à fait exact, il faut préciser que les espaces $L^{p}$ sont en réalité des espaces de classes de fonctions modulo l'égalité presque partout, au sens de la mesure de Lebesgue. En fait, en considérant uniquement un espace de fonctions, $\|\cdot\|_{L^{p}(A)}$ ne serait pas une norme et seul le passage au quotient permet de lever cet inconvénient. En procédant de la sorte, on enrichit les propriétés topologiques à disposition, notamment en faisant de $L^{p}(A)$ un espace de Banach. Cela étant, par abus de langage communément accepté, on parle tout de même de fonctions appartenant à un espace $L^{p}$ et on s'autorise à dire que $\|f\|_{L^{p}(A)}$ est la norme de $f$ et non de sa classe.

L'idée suivie pour définir les espaces de Hölder généralisés est donc, au vu de la Remarque 4, de comparer les quantités $\sup _{|h| \leq 2^{-j}}\left\|\Delta_{h}^{\lfloor\alpha\rfloor+1} f\right\|_{L^{p}\left(B_{h}\left(x_{0}, 2^{-j}\right)\right)}$ et $\sigma_{j}$, où $\boldsymbol{\sigma}$ est une suite admissible. 
Plus exactement, comme nous l'avons vu, l'appartenance à l'espace $C^{\alpha}\left(x_{0}\right)$ équivaut à la bornitude de la suite $\left(2^{\alpha j} \sup _{|h| \leq 2^{-j}}\left\|\Delta_{h}^{\lfloor\alpha\rfloor+1} f\right\|_{L^{\infty}\left(B_{h}\left(x_{0}, 2^{-j}\right)\right)}\right)_{j \in \mathbb{N}^{*}}$. En terme d'espaces fonctionnels, cela revient à demander que cette dernière appartienne à $\ell^{\infty}$. Pour gagner à nouveau en précision sur le comportement de la fonction analysée autour du point $x_{0}$, on peut alors faire intervenir les espaces $\ell^{q}$.

Définition 10. Soit $1 \leq q<\infty$. Une suite $\left(x_{j}\right)_{j}$ appartient à l'espace $\ell^{q}$ si $\sum_{j}\left|x_{j}\right|^{q}<\infty$. Dans ce cas, la norme $\ell^{q}$ de $\left(x_{j}\right)_{j}$ est définie par

$$
\left\|\left(x_{j}\right)_{j}\right\|_{\ell^{q}}:=\left(\sum_{j=1}^{+\infty}\left|x_{j}\right|^{q}\right)^{\frac{1}{q}} .
$$

Une suite $\left(x_{j}\right)_{j \in \mathbb{N}^{*}}$ appartient à l'espace $\ell^{\infty}$ si elle est bornée. Dans ce cas, la norme $\ell^{\infty}$ de $\left(x_{j}\right)_{j \in \mathbb{N}^{*}}$ est définie par

$$
\left\|\left(x_{j}\right)_{j}\right\|_{\ell^{\infty}}:=\sup _{j}\left|x_{j}\right| \text {. }
$$

Nous avons dès à présent tous les ingrédients nécessaires pour donner la définition des espaces de Hölder généralisés que nous avons proposée dans [34].

Définition 11. Soient $p, q \in[1, \infty], f \in L_{\text {loc }}^{p}, \boldsymbol{\sigma}$ une suite admissible telle que $\underline{s}(\boldsymbol{\sigma})>-\frac{-d}{p}$ et $x_{0} \in \mathbb{R}^{d} ; f$ appartient à l'espace $T_{p, q}^{\sigma}\left(x_{0}\right)$ dès lors que

$$
\left(\sigma_{j} 2^{j d / p} \sup _{|h| \leq 2^{-j}}\left\|\Delta_{h}^{\lfloor\bar{s}(\sigma)\rfloor+1} f\right\|_{L^{p}\left(B_{h}\left(x_{0}, 2^{-j}\right)\right)}\right)_{j} \in \ell^{q}
$$

Évidemment, si $p=q=\infty$ et $\boldsymbol{\sigma}$ est la suite dyadique $\left(2^{\alpha j}\right)_{j}$, on a bien $T_{p, q}^{\sigma}\left(x_{0}\right)=C^{\alpha}\left(x_{0}\right)$.

Remarque 12. Le coefficient $2^{j d / p}$ qui apparaît dans la définition précédente correspond à la norme $L^{p}$ de la fonction indicatrice 9 de $B\left(x_{0}, 2^{-j}\right)$. Il est ajouté de manière à ce que la mesure de la boule n'interfère pas dans le calcul de la régularité. En particulier, si $f$ est « la fonction de référence $\gg\left|x-x_{0}\right|^{\alpha}\left(\right.$ avec $\left.\alpha>-\frac{d}{p}\right)$, on a bien, pour tout $j \in \mathbb{N}^{*}$,

$$
2^{j d / p} \sup _{|h| \leq 2^{-j}}\left\|\Delta_{h}^{\lfloor\bar{s}(\sigma)\rfloor+1} f\right\|_{L^{p}\left(B_{h}\left(x_{0}, 2^{-j}\right)\right)}=2^{-\alpha j} .
$$

La Définition 11 doit être mise en parallèle avec celle des espaces de Besov généralisés, donnée dans [39].

Définition 13. Soient $p, q \in[1, \infty], \boldsymbol{\sigma}$ et $\boldsymbol{\gamma}$ sont deux suites admissibles telles que $\underline{\gamma}_{1}>1$ et $0<\underline{s}((\sigma)) \bar{s}(\boldsymbol{\gamma})$. Pour tout $n \in \mathbb{N}$ tel que $\bar{s}(\boldsymbol{\sigma}) \underline{s}(\boldsymbol{\gamma})<$

9. Si $A \subset \mathbb{R}^{d}$, la fonction indicatrice de $A$ est la fonction qui vaut 1 sur $A$ et 0 ailleurs. 
$n$, une fonction $f \in L^{p}\left(\mathbb{R}^{d}\right)$ appartient à l'espace de Besov généralisé $B_{p, q}^{\sigma, \gamma}$ dès lors que

$$
\left(\sigma_{j} \sup _{|h| \leq \gamma_{j}^{-1}}\left\|\Delta_{h}^{n} f\right\|_{L^{p}\left(\mathbb{R}^{d}\right)}\right)_{j} \in \ell^{q} .
$$

Ainsi, les espaces de Besov sont un outil pour comprendre le comportement global de la fonction $f$, vu que la norme $L^{p}$ est calculée sur tout $\mathbb{R}^{d}$. Les espaces $T_{p, q}^{\sigma}\left(x_{0}\right)$ sont donc les versions ponctuelles des Besov, tout comme $C^{\alpha}\left(x_{0}\right)$ est la version ponctuelle de $C^{\alpha}\left(\mathbb{R}^{d}\right)$, en prenant, pour tout $j \in \mathbb{N}, \gamma_{j}=2^{j}$. Ce dernier choix n'est pas restrictif puisque, si $\boldsymbol{\sigma}$ et $\boldsymbol{\gamma}$ sont deux suites admissibles, on peut toujours construire une suite admissible $\boldsymbol{\sigma}^{\prime}$ à partir de $\boldsymbol{\sigma}$ et $\boldsymbol{\gamma}$ de sorte que $B_{p, q}^{\boldsymbol{\sigma}, \boldsymbol{\gamma}}=B_{p, q}^{\boldsymbol{\sigma}^{\prime},\left(2^{j}\right)_{j}}$ (voir [9]). Cependant, en procédant de la sorte, les indices de Boyd de la suite $\boldsymbol{\sigma}^{\prime}$ sont plus difficilement calculables que ceux des suites $\boldsymbol{\sigma}$ et $\boldsymbol{\gamma}$ séparément. Selon le contexte, l'utilisation de deux suites admissibles reste donc profitable.

Remarque 14. Au vu de leur définition utilisant des normes $L^{p}$, les espaces $T_{p, q}^{\sigma}\left(x_{0}\right)$ et $B_{p, q}^{\sigma, \gamma}$ n'échappent pas ${ }^{10}$ à la Remarque 9. À nouveau, ce passage au quotient qu'on s'autorise à ne pas mentionner permet d'obtenir des propriétés topologiques plus riches puisque $T_{p, q}^{\sigma}\left(x_{0}\right)$ et $B_{p, q}^{\sigma, \gamma}$ munis de leur norme naturelle respective

$$
\|\cdot\|_{T_{p, q}^{\sigma}\left(x_{0}\right)}: f \mapsto\|f\|_{L^{p}\left(B\left(x_{0}, 1\right)\right.}+\left\|\left(\sigma_{j} 2^{j d / p} \sup _{|h| \leq 2^{-j}}\left\|\Delta_{h}^{\lfloor\bar{s}(\sigma)\rfloor+1} f\right\|_{L^{p}\left(B_{h}\left(x_{0}, 2^{-j}\right)\right)}\right)_{j}\right\|_{\ell^{q}}
$$

et

$$
\|\cdot\|_{B_{p, q}^{\sigma, \gamma}}: f \mapsto\left\|\left(\sigma_{j} \sup _{|h| \leq \gamma_{j}^{-1}}\left\|\Delta_{h}^{n} f\right\|_{L^{p}\left(\mathbb{R}^{d}\right)}\right){ }_{j}\right\|_{\ell^{q}}
$$

sont également des espaces de Banach. On peut alors, par exemple, montrer que les fonctions infiniment continûment dérivables à support compact sont denses dans $\left(T_{p, q}^{\boldsymbol{\sigma}}\left(x_{0}\right),\|\cdot\|_{T_{p, q}^{\sigma}\left(x_{0}\right)}\right)$ et en déduire qu'il s'agit d'espaces séparables [33].

Dans [32, nous avons obtenu diverses caractérisations des espaces de Besov généralisés en terme de différentiabilité, approximation polynomiale, convolution et interpolation, et ce afin de mieux comprendre ceux-ci et l'information qu'ils apportent sur la régularité (uniforme) d'une fonction. Nous allons voir qu'ils apportent également une riche information sur la régularité ponctuelle d'une fonction.

Dans les sections suivantes, afin d'alléger les notations, si, pour tout $j \in \mathbb{N}, \gamma_{j}=2^{j}$, nous noterons l'espace de Besov $B_{p, q}^{\sigma}$ au lieu de $B_{p, q}^{\boldsymbol{\sigma}, \boldsymbol{\gamma}}$. $\mathrm{Si}$, de plus, il existe $\alpha \in \mathbb{R}$ tel que, pour tout $j \in \mathbb{N}, \sigma_{j}=2^{\alpha j}$, nous désignerons l'espace de Besov associé $B_{p, q}^{\alpha}$.

10. Ainsi, modifier une fonction en un ensemble négligeable de points n'altère pas la régularité mesurée par les espaces fonctionnels que nous considérons ici. 


\section{Ondelettes}

Dans cette section, nous allons montrer comment la régularité ponctuelle au sens des espaces $T_{p, q}^{\boldsymbol{\sigma}}\left(x_{0}\right)$ s'exprime sous la forme de conditions sur la décomposition en une base d'ondelettes. Il est intéressant de mentionner que l'étude de la régularité au travers d'une base de fonctions est une question récurrente en mathématique. On peut par exemple citer les travaux de Ciesielski [10, 18] qui transcrit la régularité höldérienne sur $[0,1]$ via la base de Faber-Schauder construite à partir de la fonction triangulaire

$$
\Delta(t)= \begin{cases}2 t & \text { si } 0 \leq t \leq \frac{1}{2} \\ 2(1-t) & \text { si } \frac{1}{2}<t \leq 1 \\ 0 & \text { sinon }\end{cases}
$$

Ces dernières années, les bases d'ondelettes sont privilégiées car elles présentent de nombreux avantages, aussi bien théoriques que pratiques : elles peuvent être de régularité arbitraire 11 , elles sont bien localisées en temps et en fréquence, elles sont faciles à utiliser numériquement,...

Sous certaines hypothèses assez générales (pour plus de précisions voir [12, 35, 38]) portant sur la fonction $\varphi$ et les $2^{d}-1$ fonctions $\left(\psi^{(l)}\right)_{1 \leq l<2^{d}}$, appelées ondelettes, la famille

$$
\left\{\varphi(x-k): k \in \mathbb{Z}^{d}\right\} \cup\left\{\psi^{(l)}\left(2^{j} x-k\right): 1 \leq l<2^{d}, k \in \mathbb{Z}^{d}, j \in \mathbb{N}\right\}
$$

forme une base orthonormée de $L^{2}\left(\mathbb{R}^{d}\right)$. Cela signifie en particulier que n'importe quelle fonction $f \in L^{2}\left(\mathbb{R}^{d}\right)$ peut être décomposée de la sorte :

$$
f(x)=\sum_{k \in \mathbb{Z}^{d}} C_{k} \varphi(x-k)+\sum_{j \in \mathbb{N}} \sum_{k \in \mathbb{Z}^{d}} \sum_{1 \leq l<2^{d}} c_{j, k}^{(l)} \psi^{(l)}\left(2^{j} x-k\right),
$$

où

et

$$
c_{j, k}^{(l)}=2^{d j} \int_{\mathbb{R}^{d}} f(x) \psi^{(l)}\left(2^{j} x-k\right) d x
$$

$$
C_{k}=\int_{\mathbb{R}^{d}} f(x) \varphi(x-k) d x .
$$

En pratique, on considère essentiellement deux familles majeures d'ondelettes :

— les ondelettes de Daubechies, à support compact [12] ;

- les ondelettes de Lemarié-Meyer, à décroissance rapide [30].

Un dilaté-translaté d'ondelettes $\psi^{(l)}\left(2^{j} x-k\right)$ est centré autour du cube dyadique

$$
\lambda_{j, k}^{(l)}:=\frac{l}{2^{j+1}}+\frac{k}{2^{j}}+\left[0, \frac{1}{2^{j+1}}\left[^{d},\right.\right.
$$

11. Cela permet de ne pas « voir » la régularité de l'ondelette dans nos calculs et uniquement tirer des conclusions sur celle de la fonction analysée 
à tel point que, en omettant les indices $j, k, l$, on préfère noter $\psi_{\lambda}$ (resp. $c_{\lambda}$ ), le dilaté-translaté d'ondelette (resp. le coefficient d'ondelette) associé au cube $\lambda$. On dit que $j$ est l'échelle du cube dyadique $\lambda_{j, k}^{(l)}$ et on note $\Lambda_{j}$ l'ensemble des cubes dyadiques d'échelle $j$.

Les ondelettes nous offrent donc une sorte de microscope sur la fonction analysée $f$, en la décomposant en une série d'ondelettes. De nombreux espaces fonctionnels admettent alors une caractérisation par les coefficients en ondelettes, sans pour autant devoir demander des hypothèses supplémentaires sur la base d'ondelette [38. Par exemple, si $p, q \in[1,+\infty]$ et $\boldsymbol{\sigma}$ est une suite admissible, une fonction appartient à $B_{p, q}^{\sigma}$ si et seulement si la suite $\left(C_{k}\right)_{k}$ définie par (7) appartient à $\ell^{q}$ et si 12

$$
\left(\sum_{j \in \mathbb{N}}\left(\sum_{\lambda \in \Lambda_{j}}\left(2^{-j d / p} \sigma_{j}\left|c_{\lambda}\right|\right)^{p}\right)^{\frac{q}{p}}<\infty .\right.
$$

L'idée est alors que la régularité ponctuelle en un point $x_{0}$ se traduit dans le comportement des coefficients associés à des cubes dyadiques situés près de $x_{0}$. Définissons dans ce but les $p$-coefficients dominants d'ondelettes. Pour ce faire, précisons que, si $\lambda$ est un cube dyadique, $3 \lambda$ dénote l'ensemble formé par $\lambda$ et ses $3^{d}-1$ cubes adjacents. Si $x_{0} \in \mathbb{R}^{d}$ et $j \in \mathbb{N}, \lambda_{j}\left(x_{0}\right)$ est l'unique cube dyadique d'échelle $j$ qui contient $x_{0}$.

Définition 15. Soient $p \in[1, \infty], j \in \mathbb{N}$ et $x_{0} \in \mathbb{R}^{d}$. Étant donné un cube dyadique $\lambda \in \Lambda_{j}$, on note

$$
d_{\lambda}^{p}=\sup _{j^{\prime} \geq j}\left(\sum_{\lambda^{\prime} \in \Lambda_{j^{\prime}}, \lambda^{\prime} \subset \lambda}\left(2^{\left(j-j^{\prime}\right) d / p}\left|c_{\lambda^{\prime}}\right|\right)^{p}\right)^{1 / p} .
$$

Le p-coefficient dominant d'échelle $j$ en $x_{0}$ est alors défini par

$$
d_{j}^{p}\left(x_{0}\right)=\sup _{\lambda \in 3 \lambda_{j}\left(x_{0}\right)} d_{\lambda}^{p}
$$

Dans [34], nous utilisons les $p$-coefficients dominants pour extraire des informations sur la régularité ponctuelle. Nous avons obtenu le résultat suivant, qui est valable aussi bien pour les ondelettes de Daubechies que pour les ondelettes de Lemarié-Meyer.

Théorème 16. Si la fonction $f$ appartient à l'espace $T_{p, q}^{\sigma}\left(x_{0}\right)$ alors

$$
\left(\sigma_{j} d_{j}^{p}\left(x_{0}\right)\right)_{j} \in \ell^{q}
$$

12. Si $p=\infty$ (resp. $q=\infty)$, on adopte la convention que la somme sur $\lambda \in \Lambda_{j}$ (resp. $j \in \mathbb{N}$ ) est remplacée par la borne supérieure sur le même ensemble. 
Réciproquement, si la suite admissible $\boldsymbol{\sigma}$ est telle que $2^{-j d / p} \sigma_{j}^{-1} \rightarrow 0$ et $\underline{\sigma}_{1}>2^{-d / p}$ et s'il existe $\varepsilon>0$ tel que $f \in B_{p, q}^{\varepsilon}$ alors $f \in T_{p, q}^{\sigma_{\log }}\left(x_{0}\right)$, où $\boldsymbol{\sigma}_{\log }$ désigne la suite admissible définie, pour tout $j \in \mathbb{N}$, par

$$
\left(\boldsymbol{\sigma}_{\log }\right)_{j}=\frac{\sigma_{j}}{\left|\log _{2}\left(2^{-j d / p} \sigma_{j}^{-1}\right)\right|} .
$$

Le théorème nous indique donc que les ondelettes nous donnent une quasi-caractérisation de la régularité ponctuelle, au sens des espaces $T_{p, q}^{\sigma}\left(x_{0}\right)$, ce qui permet de réduire son étude à des calculs de coefficients, souvent bien plus accessibles que la définition brute. Arrêtons-nous un instant sur la réciproque.

Premièrement, les hypothèses faites sur $\boldsymbol{\sigma}$, nécessaires pour notre démonstration, ne sont pas lourdes, facilement vérifiables et correspondent bien à une situation pratique car pour avoir une étude assez significative de la régularité d'une fonction, on préfère toujours travailler avec des suites à croissance suffisamment importante.

En ce qui concerne l'appartenance à un espace de Besov uniforme, également nécessaire dans notre démonstration, il semble naturel de demander qu'une fonction ait une régularité uniforme minimum pour pouvoir lui appliquer nos outils d'analyse et ainsi en extraire une information exacte sur sa régularité ponctuelle. De plus, dans [22], l'auteur montre que, dans le cadre des espaces $C^{\alpha}\left(x_{0}\right)$, cette condition est optimale, à savoir que, sans l'hypothèse de régularité uniforme, on peut construire une fonction vérifiant la condition demandée sur les coefficients en ondelettes mais n'appartenant pas à l'espace fonctionnel proposé.

Finalement, en ce qui concerne la correction logarithmique, dans le cadre d'un papier en préparation, nous avons montré que le résultat est également optimal. En effet, si $\boldsymbol{\sigma}$ est une suite admissible qui satisfait aux hypothèses du Théorème 16, nous avons construit (voir [31]) une fonction $f \in B_{\infty, \infty}^{\varepsilon}$, pour un $\varepsilon>0$, telle que

$$
\left(\sigma_{j} d_{j}^{\infty}(0)\right)_{j} \in \ell^{\infty}
$$

mais pour laquelle

$$
\left|\Delta_{2^{-2^{m-1}}}^{\lfloor\bar{s}(\boldsymbol{\sigma})\rfloor+1} f(0)\right|=C 2^{m} \sigma_{2^{m-1}}^{-1} \geq C^{\prime}\left|\log \left(\sigma_{2^{m-1}}\right)\right| \sigma_{2^{m-1}}^{-1},
$$

ce qui montre que $f \notin T_{\infty, \infty}^{\sigma}\left(x_{0}\right)$ et même que, pour toute fonction $g$ asymptotiquement plus faible que le logarithme, $\left(g\left(\sigma_{j}\right)\right)^{-1}$ ne peut pas être la correction apportée lors de la seconde partie du Théorème 16.

Cet exemple montre donc que, lorsqu'on étudie la régularité ponctuelle, les ondelettes ne peuvent pas caractériser exactement l'espace, vu l'apparition de cette correction logarithmique. Cependant, lorsqu'on cherche à déterminer un exposant de Hölder (généralisé), celui-ci ne sera pas, sous des hypothèses légères sur les suites admissibles, sensible 
à cette correction et l'outil ondelette reste performant, comme nous allons le voir.

\section{Analyse multifractale}

Afin de pouvoir définir une notion d'exposants de Hölder, il nous faut comme pour les espaces $C^{\alpha}\left(x_{0}\right)$ travailler avec des espaces emboîtés. Puisqu'à priori il n'existe pas de lien direct entre deux suites admissibles, c'est à nous d'imposer cette hypothèse via la définition suivante.

Définition 17. Soient $p, q \in[1, \infty]$; si, étant donné $h>-d / p, \gamma^{(h)}$ est une suite admissible, la famille de suites admissibles $h \mapsto \gamma^{(h)}$ est $(p, q)$-décroissante si elle satisfait $\underline{s}\left(\gamma^{(h)}\right)>-d / p, \underline{\gamma}_{1}^{(h)}>2^{-d / p}$ pour tout $h>-d / p$ et si $-d / p<h<h^{\prime}$ implique

$$
T_{p, q}^{\gamma^{(h)}}\left(x_{0}\right) \subset T_{p, q}^{\gamma^{\left(h^{\prime}\right)}}\left(x_{0}\right) .
$$

En pratique, ces conditions sont facilement vérifiables vu que, pour rappel, nous travaillons avec des suites de la forme $\left(\gamma_{j}^{(h)}=2^{j h} \delta_{j}^{(h)}\right)_{j}$ où $\left(\delta_{j}^{(h)}\right)_{j}$ est une suite admissible asymptotiquement plus faible que $\left(2^{j h}\right)_{j}$. Il suffit alors que les indices de Boyd des suites $\left(\delta_{j}^{(h)}\right)_{j}$ varient moins vite que $h$ pour assurer la décroissance des espaces. De manière plus générale, des conditions pour qu'une famille de suites admissibles soit décroissante ont été données dans [29].

Si $\gamma^{(h)}$ est une famille $(p, q)$-décroissante de suites admissibles, on peut alors définir un exposant de Hölder généralisé associé à cette famille.

Définition 18. Étant donnés $p, q \in[1, \infty]$ et une famille décroissante de suites admissibles $\gamma^{(\cdot)}$. Le $(p, q)$-exposant de Hölder généralisé, associé à une fonction $f \in L_{\mathrm{loc}}^{p}\left(\mathbb{R}^{d}\right)$ et $\gamma^{(\cdot)}$ en $x_{0} \in \mathbb{R}^{d}$ est défini par

$$
h_{p, q}\left(x_{0}\right):=\sup \left\{h>-d / p: f \in T_{p, q}^{\gamma^{(h)}}\left(x_{0}\right)\right\} .
$$

Fixons dès à présent une famille décroissante de suites admissibles $\gamma^{(\cdot)}$. Pour une fonction irrégulière, l'exposant de Hölder (généralisé) varie très fortement d'un point à un autre et la fonction $x_{0} \mapsto h_{p, q}\left(x_{0}\right)$ est elle-même extrêmement irrégulière. Dès lors que $h_{p, q}$ prend deux valeurs différentes, on parle de fonction multifractale. Sinon, la fonction est dite monofractale.

Puisqu'il serait alors inutile de remplacer l'étude d'une fonction irrégulière par celle d'une autre, on préfère étudier le spectre des fonctions multifractales. Afin d'introduire cette notion, nous avons besoin de définir la dimension de Hausdorff d'un ensemble.

Définition 19. Soit $A$ un sous-ensemble de $\mathbb{R}^{d}$ et $h \geq 0$. Si $\varepsilon>0$, on dit que $\left(A_{j}\right)_{j}$ est un recouvrement de $A$ subordonné à $\varepsilon$ de $A$, ou 
encore un $\varepsilon$-recouvrement de $A$, si $A \subseteq \bigcup_{j} A_{j}$ et, pour tout $j$, on a ${ }^{13}$ $\operatorname{diam}\left(A_{j}\right) \leq \varepsilon$. La mesure $h$ de Hausdorff de $A$ est définie par

$\mathcal{H}^{h}(A):=\lim _{\varepsilon \rightarrow 0^{+}} \inf \left\{\sum_{j} \operatorname{diam}\left(A_{j}\right)^{h}:\left(A_{j}\right)_{j}\right.$ est un $\varepsilon$-recouvrement de $\left.A\right\}$.

On peut alors montrer que la fonction $h \mapsto \mathcal{H}^{h}(A)$ admet une unique valeur $h_{0}$ pour laquelle, pour tout $h<h_{0}, \mathcal{H}^{h}(A)=\infty$ et pour tout $h>h_{0}, \mathcal{H}^{h}(A)=0$. La mesure de Hausdorff fait donc un « saut » en cette valeur critique qui est définie comme étant la dimension de Hausdorff de $A$. Formellement, si $4 \neq \emptyset$, on a

$$
\operatorname{dim}_{\mathcal{H}}(A)=\sup \left\{h \geq 0: \mathcal{H}^{h}(A)=\infty\right\}=\inf \left\{h \geq 0: \mathcal{H}^{h}(A)=0\right\} .
$$

Dans le même esprit que l'exposant de Hölder qui vise à donner un continuum de valeurs à la mesure de régularité, la dimension de Hausdorff cherche à donner un continuum de valeurs pour la dimension, grandeur qui renseigne sur «l'épaisseur» d'un objet dans l'espace. Elle prolonge bien la notion intuitive de dimension puisque la dimension de Hausdorff d'un ouvert de $\mathbb{R}^{d}$ est $d$.

Le $(p, q)$-spectre associé à la famille $\gamma^{(\cdot)}$ d'une fonction $f \in L_{\text {loc }}^{p}\left(\mathbb{R}^{d}\right)$ est alors défini par

$$
D_{p, q}(h):=\operatorname{dim}_{\mathcal{H}}\left\{x_{0} \in \mathbb{R}^{d}: h_{p, q}\left(x_{0}\right)=h\right\} .
$$

Remarquons que le spectre associé à l'exposant de Hölder classique, que nous notons $D(h)$, permet déjà de différencier la régularité de la fonction de Weierstraß qui est monofractale de celle de la fonction de Riemann qui est multifractale.

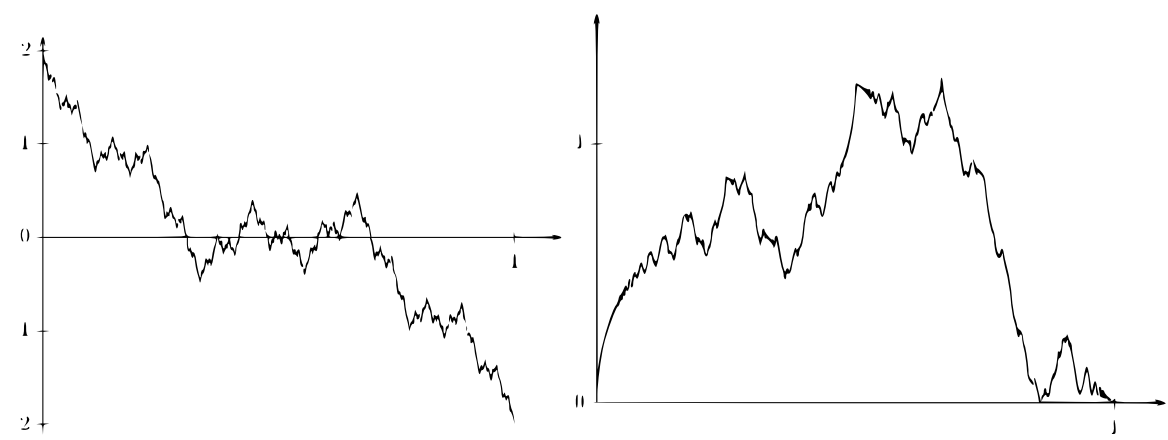

Figure 5. Représentations graphiques (issues de [15] ) de la fonction de Weierstraß de paramètres $a=\frac{1}{2}$ et $b=3$ et de la fonction de Riemann.

Comme mentionné ci-dessus, le comportement de la fonction de Weierstrass est identique en tout point, c'est-à-dire que, pour tout $x_{0} \in \mathbb{R}$,

13. Le diamètre de $A$ est défini par $\operatorname{diam}(A):=\sup _{x, y \in A}|x-y|$

14. La dimension de Hausdorff de l'ensemble vide est $-\infty$ par convention. 
$h_{\mathcal{W}_{a, b}}\left(x_{0}\right)=-\log (a) / \log (b)$, ce qui donne, en terme de spectre,

$$
D(h)= \begin{cases}1 & \text { si } h=-\frac{\log (a)}{\log (b)} \\ -\infty & \text { sinon }\end{cases}
$$

tandis que, concernant la fonction de Riemann, beaucoup plus irrégulières en certains points que d'autres, on a

$$
D(h)= \begin{cases}4 h-2 & \text { si } h \in\left[\frac{1}{2}, \frac{3}{4}\right] \\ 0 & \text { si } h=\frac{3}{2} \\ -\infty & \text { sinon. }\end{cases}
$$

Ce spectre a été établi par Stéphane Jaffard dans [24] .

Un formalisme multifractal est une méthode, voire même une formule, qui vise à associer à une fonction les valeurs de son spectre, sous certaines conditions, c'est-à-dire sous l'hypothèse d'appartenance à un espace fonctionnel. Un moyen de juger la validité d'un formalisme est d'utiliser la notion de prévalence. Précédemment, dans $\mathbb{R}^{d}$, nous avons expliqué que la mesure de Lebesgue permet de définir proprement l'idée de «presque partout». Malheureusement toutes les riches propriétés de la mesure de Lebesgue ne peuvent être exportées dans des espaces de fonctions, qui sont de dimension infinie. L'idée de la prévalence est de transposer certaines des caractéristiques des ensembles de mesure de Lebesgue nulle en définition afin d'obtenir une notion similaire dans ce cadre plus général. Une étude détaillée de la prévalence est trop lourde pour être abordée dans ce papier mais le lecteur intéressé peut consulter [21], l'essentiel ici est de retenir que la prévalence permet, dans une approche probabiliste, de dire si un ensemble est important ou non.

Dans [34], nous avons proposé le formalisme multifractal suivant pour exprimer le spectre $D_{p, q}$ d'une fonction appartenant à un espace de Besov $B_{r, s}^{\sigma}$ :

$$
D_{p, q}(h)=d+r \bar{s}\left(\frac{\boldsymbol{\gamma}^{(h)}}{\boldsymbol{\sigma}}\right) .
$$

Pour juger de sa validité, nous considérons une suite $\boldsymbol{\sigma}$ dite compatible avec la famille $\gamma^{(\cdot)}$.

Définition 20. Une suite admissible $\boldsymbol{\sigma}$ et une famille de suites admissibles $\gamma^{(\cdot)}$ sont compatibles pour $p, q, r, s \in[1, \infty]$ avec $s \leq q$ si

$-\underline{s}(\boldsymbol{\sigma})>0$,

$-\underline{s}(\boldsymbol{\sigma})-d / r>-d / p$,

- la fonction $\zeta$ définie sur $(-d / p, \infty)$ par

$$
\zeta(h):=\underline{s}\left(\frac{\boldsymbol{\gamma}^{(h)}}{\boldsymbol{\sigma}}\right)=\bar{s}\left(\frac{\boldsymbol{\gamma}^{(h)}}{\boldsymbol{\sigma}}\right)
$$

est croissante, continue et telle que

$$
\{h>-d / p: \zeta(h)<-d / r\} \neq \emptyset .
$$


Bulletin de la Société Royale des Sciences, Vol. 90, Articles, 2021, p. 49 - 71

On appelle $\zeta$ la fonction ratio. Nous utiliserons également la quantité

$$
h_{\min }(r):=\sup \{h>-d / p: \zeta(h)<-d / r\} .
$$

L'idée principale de la condition de compatibilité, traduite par (10), est que la suite $\boldsymbol{\sigma}$ compense suffisamment la partie non dyadique des suites $\gamma^{(h)}$ de manière à ce que les indices de Boyd de $\frac{\gamma^{(h)}}{\sigma}$ soient identiques. De cette manière, pour tout $h>-\frac{d}{p}$, si $\kappa^{(h)}$ est la partie non dyadique de $\frac{\gamma^{(h)}}{\sigma}$, pour tout $\varepsilon>0$, il existe $C>0$ tel que, pour tout $j \in \mathbb{N}$,

$$
C^{-1} 2^{-\varepsilon j} \leq \kappa_{j}^{(h)} \leq C 2^{\varepsilon j}
$$

Afin de tirer parti de la caractérisation en ondelettes développée à la section précédente, nous avons alors remarqué que, si $p, r, s \in[1,+\infty]$ et $\boldsymbol{\sigma}$ est une suite admissible telle que $\underline{s}(\boldsymbol{\sigma})>0$ et $\underline{s}(\boldsymbol{\sigma})-\frac{d}{r}>-\frac{d}{p}$, une fonction $f$ appartient à $B_{r, s}^{\sigma}$ si et seulement si la suite $\left(C_{k}\right)_{k}$ définie par (7) appartient à $\ell^{s}$ et si

$$
\left(\sum_{j \in \mathbb{N}}\left(\sum_{\lambda \in \Lambda_{j}}\left(\sigma_{j} 2^{-d j / r} d_{\lambda}^{p}\right)^{r}\right)^{s / r}\right)^{1 / s}<\infty .
$$

À l'aide du Théorème 16, on peut valider le formalisme multifractal proposé. Les détails des démonstrations de ces derniers résultats peuvent à nouveau être consultés dans [34].

Théorème 21. Soient $p, q, r, s \in[1, \infty]$ avec $s \leq q, \boldsymbol{\sigma}$ une suite admissible et $\boldsymbol{\gamma}^{(\cdot)}$ une famille de suites admissibles compatible avec $\boldsymbol{\sigma}$. D'un point de vue prévalent, pour presque toute fonction $f \in B_{r, s}^{\sigma}$, le spectre $D_{p, q}$ est défini sur $I=\left[\zeta^{-1}(-d / r), \zeta^{-1}(0)\right]$ et

$$
D_{p, q}(h)=d+r \zeta(h),
$$

pour tout $h \in I$.

De plus, pour presque tout $x_{0} \in \mathbb{R}^{d}$, on a $h_{p, q}\left(x_{0}\right)=\zeta^{-1}(0)$.

Ce dernier théorème nous offre ainsi la possibilité d'étudier assez rapidement le spectre d'une fonction appartenant à un espace de Besov généralisé. De plus, il affirme également que, pour presque toute fonction dans ce dernier, la régularité ponctuelle la plus répandue est celle la plus proche de la suite admissible qui définit l'espace de Besov, c'est-à-dire le $h$ pour lequel $\zeta(h)=0$.

En implémentant des méthodes numériques basées sur ces résultats, il semble, statistiquement, que nous sommes capables de différencier un mouvement brownien d'une autre fonction. Dans [26, la méthode expérimentale consiste à mesurer l'exposant de Hölder du mouvement brownien et d'une version aléatoire de la fonction de Weierstraß :

$$
W(x)=\sum_{j=0} a^{j} \cos \left(\left(b^{j} x+U_{j}\right) \pi\right),
$$


où $\left(U_{j}\right)_{j}$ est une suite de variables aléatoires indépendantes de même loi uniforme sur $[0,1]$ et $0<a<1<b$ sont choisis de telle sorte que $-\log (a) / \log (b)=\frac{1}{2}$. Ainsi, presque sûrement, $W$ est d'exposant de Hölder $\frac{1}{2}$ et appartient à $C^{\frac{1}{2}}(\mathbb{R})$. Pour rappel, le mouvement brownien présente une correction logarithmique itérée dans son comportement ponctuel. L'idée est alors d'appliquer successivement divers algorithmes de calculs de régularité à 20 réalisations générées aléatoirement, via le calcul de $2^{20}$ coefficients en ondelettes, du mouvement brownien et de la fonction $W$. La seule variante entre les algorithmes est la famille de suites admissibles qui leur est associée : le premier repose sur la famille $\left(\left(2^{\alpha j}\right)_{j}\right)_{\alpha>0}$, le second repose sur $\left(\left(2^{\alpha j}|\log j|^{-\frac{1}{2}}\right)_{j}\right)_{\alpha>0}$, le troisième repose sur $\left(\left(2^{\alpha j}|\log j|^{-1}\right)_{j}\right)_{\alpha>0}$ et finalement le quatrième sur $\left(\left(2^{\alpha j} j^{-\frac{1}{2}}\right)_{j}\right)_{\alpha>0}$. Nous savons que les deux fonctions ont, presque sûrement leur exposant de Hölder en tous points égal à 1/2. Les boites à moustaches présentées à la Figure 5 reprennent les erreurs entre l'exposant mesuré par les quatre algorithmes lors de leurs exécutions sur les 20 réalisations de chaque fonction et $\frac{1}{2}$.
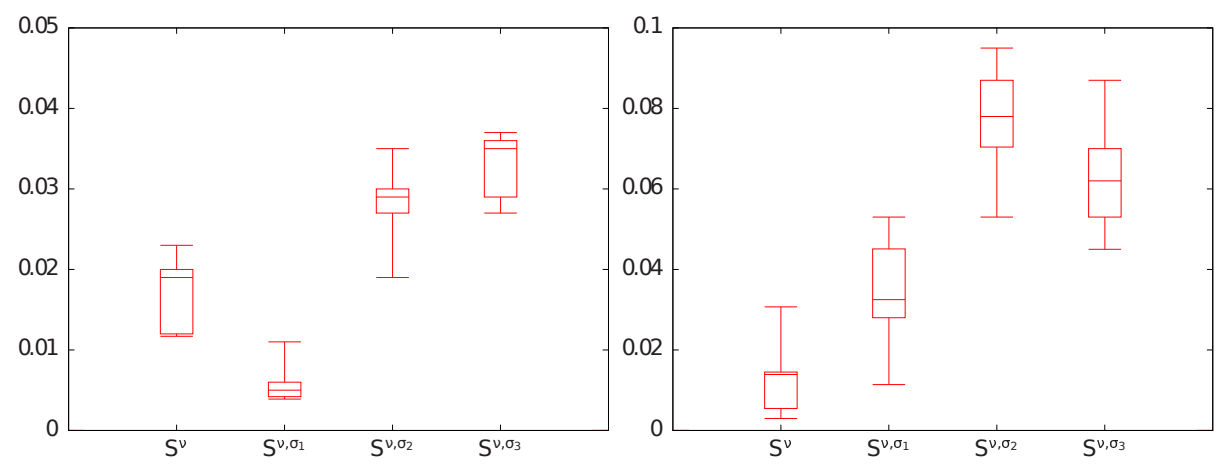

Figure 6. Boîtes à moustaches reprenant l'erreur de mesure de l'exposant de Hölder du mouvement brownien (à gauche) et de la fonction de Weierstraß aléatoire (à droite) lors de l'exécution de l'algorithme associé aux familles $\left(2^{\alpha j}\right)_{j}, \boldsymbol{\sigma}_{1}^{(\boldsymbol{\alpha})}=\left(2^{\alpha j}|\log j|^{-\frac{1}{2}}\right)_{j}, \boldsymbol{\sigma}_{\mathbf{2}}^{(\boldsymbol{\alpha})}=$ $\left(2^{\alpha j}|\log j|^{-1}\right)_{j}$ et $\boldsymbol{\sigma}_{\mathbf{3}}^{(\boldsymbol{\alpha})}=\left(2^{\alpha j} j^{-\frac{1}{2}}\right)_{j}$ sur 20 échantillons de chaque fonction

Nous constatons alors que l'algorithme produisant statistiquement le moins d'erreur pour chaque fonction est celui associé à la famille de suites admissibles qui correspond au comportement exact de la fonction, avec un logarithme itéré pour le mouvement brownien, et pas de correction logarithmique pour la fonction de Weierstrass. De plus, les boites à moustaches les moins étendues sont également celles associées à la famille appropriée. Ainsi, pour détecter la présence ou non de corrections logarithmiques dans une fonction aléatoire, nous proposons de répéter un nombre statistiquement significatif de fois sur des 
réalisations de la fonction l'exécution de l'algorithme associé à diverses familles. Celle présentant les résultats les moins dispersés rendrait alors compte de la correction présente.

En conclusion, nous avons montré que la découverte des fonctions continues mais non dérivables a abouti à la mise en œuvre d'une nouvelle théorie basée sur la notion d'exposants d'Hölder. Cela étant, les exemples d'application de cette dernière nous ont montré qu'il était bon de généraliser les outils de travail afin de pouvoir à la fois considérer plus de fonctions mais aussi de les caractériser plus finement. Grâce à la transformée en ondelettes et des espaces fonctionnels, nous proposons un formalisme précis pour étudier la régularité ponctuelle généralisée. Les applications numériques de celui-ci tendent à montrer, qu'en pratique, notre méthode permet notamment de détecter les corrections logarithmiques présentes dans le comportement ponctuel d'une fonction. Cela pourrait par exemple être appliqué dans le milieu financier puisque le modèle de Black-Scholes demande de vérifier en première hypothèse que le cours du prix des actions boursières suivent un mouvement brownien ([5, 20]).

Remerciements. Mes recherches sont financées par une bourse d'aspirant FNRS. Je remercie évidemment Samuel Nicolay pour notre collaboration quotidienne qui a abouti aux résultats exposés dans ce papier. Je remercie également Marie Lejeune et Céline Esser pour leurs avis éclairés sur la rédaction de ce dernier. Je tiens également à remercier le référé anonyme pour sa relecture attentive de la première version de cet article et ses nombreuses suggestions ayant contribué à une nette amélioration de celui-ci.

\section{RÉFÉRENCES}

[1] L.F. Abott et M.B. Wise, Dimension of quantum-mechanical path, Am. J. Phys., 49,37-39 (1981).

[2] M.V. Altaisky, L.P. Chernenko, V.M. Balebanov, N.S. Erokhin et S.S. Moiseev, Multifractal analysis of AFM images of $\mathrm{Nb}$ thin film surfaces, Particles and Nuclei, Letters, 2, 14-26 (2000).

[3] A.-M. Ampère, Recherche sur quelques points de la théorie des fonctions dérivées qui conduisent à une nouvelle démonstration du théorème de Taylor, et à l'expression finie des termes qu'on néglige lorsqu'on arrête cette série à un terme quelconque, J. Éc. polytech., 6, 148-181 (1806).

[4] A. Baouche et S. Dubuc, La non-dérivabilité de la fonction de Weierstrass, L'Enseignement des Math., 39, 84-94 (1992).

[5] F. Black et M. Scholes, The Pricing of Options and Corporate Liabilities, Journal of Political Economy, 81 3, 637-654 (1973).

[6] P. du Bois-Reymond, Versuch einer Classification der willkürlichen Functionen reeller Argumente nach ihren Aenderungen in den kleinsten Intervallen, J. Reine. Angew. Math., 79, 21-37 (1875). 
[7] Yu. A. Brudnyi, A multidimensional analog of a theorem of Whitney, Mathematics of the USSR, 11 2, 157-170 (1970).

[8] R. Brown, A brief Account of Microscopical Observations made in the Months of June, July, and August, 182\%, on the Particles contained in the Pollen of Plants; and on the general Existence of active Molecules in Organic and Inorganic Bodies, Edinburgh New Philos. J., 5, 358-371 (1828).

[9] A.M. Caetano et H.-G. Leopold, On generalized Besov and Triebel-Lizorkin spaces of regular distributions, J. Funct. Anal., 264, 266-2703 (2013).

[10] Z. Ciesielski, On the isomorphisms of the space $H_{\alpha}$ and $m$, Bull. Acad. Polon. Sci. Sér. Sci. Math. Astronom. Phys. 8, 217-222 (1960).

[11] M. Clausel et S. Nicolay, Some prevalent results about strongly monoHölder functions, Nonlinearity, 23, 2101-2116 (2010)

[12] I. Daubechies, Ten Lectures on Wavelets, CBMS-NSF Regional Conference Series in Applied Mathematics (1992).

[13] A. Deliège et S. Nicolay, Köppen-Geiger climate classification for Europe recaptured via the Hölder regularity of air temperature data, Pure Appl. Geophys.,173, 2885-2898 (2016).

[14] A. Einstein, Investigations on the Theory of the Brownian Movement, Dover Publications, inc. (1985).

[15] C. Esser, Regularity of functions : Genericity and multifractal analysis, Thèse de doctorat, Université de Liège (2014).

[16] U. Frisch et G. Parisi, On the singularity structure of fully developed turbulence; appendix to Fully developped turbulence and intermittency, Proc. Int. Summer School Phys. Enrico Fermi, 84-88 (1985).

[17] J. Gray, Plato's Ghost : The Modernist Transformation of Mathematics, Princeton University Press (2009).

[18] D. Hamadouche, Invariance principles in Hölder spaces, Portugal. Math. 57, no. 2, 127-151 (2000).

[19] G. Hardy, Weierstrass's Non-Differentiable Function, Trans. Amer. Math. Soc., 17, 301-325 (1916).

[20] E.G. Haug, Option Pricing and Hedging from Theory to Practice, Derivatives : Models on Models, Wiley (2007).

[21] B.R. Hunt, T. Sauer et J.A. Yorke, Prevalence : a translation-invariant «almost every $\gg$ on infinite-dimensional spaces, Bull. Amer. Math. Soc., 27, 217238 (1992).

[22] S. Jaffard, Pointwise smoothness, two-microlocalization and wavelet coeffcients, Publ. Mat., 35 1, 155-168 (1991).

[23] S. Jaffard et B. Martin, Multifractal anlysis of the Brjuno function. Invent. Math., 212, 109-132 (2018).

[24] S. Jaffard, The spectrum of singularities of Riemann's function, J. Math. Phys., 39 8, 4129-4141 (1998).

[25] A. Khintchine, Über einen Satz der Wahrscheinlichkeitsrechnung, Fund. Math., 6, 9-20 (1924).

[26] T. Kleyntssens, New Methods for Signal Analysis : Multifractal Formalisms based on Profiles. From Theory to Practice, Thèse de doctorat (2019).

[27] D. Kreit, On Generalized Hölder-Zygmund Spaces, Thèse de Doctorat, Université de Liège (2016). 
[28] D. Kreit et S. Nicolay, Some characterizations of generalized Hölder spaces, Math. Nachr., 285, 2157-2172 (2012).

[29] D. Kreit et S. Nicolay, Generalized pointwise Hölder spaces defined via admissible sequences, J. Funct. Spaces, 11 pages (2018).

[30] P.G. Lemarié et Y. Meyer, Ondelettes et bases hilbertiennes, Rev. Mat. Iberoam. 2, 1-18 (1986)

[31] L. Loosveldt, About some notions of regularity for functions, Thèse de doctorat, Université de Liège (2021).

[32] L. Loosveldt et S. Nicolay, Some equivalent definitions of Besov spaces of generalized smoothness, Math. Nachr, 292-10, 2262-2282 (2019).

[33] L. Loosveldt et S. Nicolay, Generalized $T_{u}^{p}$ spaces : On the trail of Calderón and Zygmund, Diss. Math, 554, 1-64 (2020).

[34] L. Loosveldt et S. Nicolay, Generalized spaces of pointwise regularity : To a general framework for the WLM, soumis à publication (2020).

[35] S. Mallat, A Wavelet Tour of Signal Processing, Academic Press (1999).

[36] B.B. Mandelbrot, Fractals and Scaling in Finance : Discontinuity, Concentration, Risk, Springer (1997).

[37] J. McCarthy, An everywhere continuous nowhere differentiable function, Amer. Math. Montly, 60, 709 (1953).

[38] Y. Meyer et D. Sallinger. Wavelets and Operators, volume 1, Cambridge University press (1995).

[39] S.D. Moura, On some characterizations of Besov spaces of generalized smoothness, Math. Nachr., 280, 1190-1199 (2007).

[40] S. Nicolay, Analyse mathématique. Fonctions définies sur une partie de la droite réelle, Éditions Ellipses (2018).

[41] S. Nicolay, Analyse de séquences ADN par la transformée en ondelettes : extraction d'informations structurelles, dynamiques et fonctionnelles, Thèse de Doctorat (2006).

[42] S. Nicolay, E.B. Brodie, M. Touchon, B. Audit, Y. d'Aubenton-Carafa, C. Thermes et A. Arneodo, Bifractality of human DNA strand-asymmetry profiles results from transcription, Phys. Rev. E, 75 (2007).

[43] J.B. Perrin. Mouvement brownien et réalité moléculaire, Annales de Chimie et de Physique, 18, 5-114 (1909)

[44] E.E. Peters, Chaos and order in Capital Markets, John Wiley \& Sons Inc. (1991).

[45] H. Triebel, Theory of Function Spaces I-III, Birkhäuser Verlag (1983).

(Laurent Loosveldt)

Université de Liège, Département de mathématique - zone Polytech 1,

12 allée de la Découverte, BÂt. B37, B-4000 Liège

Email address: 1.loosveldt@uliege.be 